\title{
Jean Piaget Society
}

Society for the Study of Knowledge and Development

The Jean Piaget Society for the Study of Knowledge and Development (JPS) is delighted to partner with Karger to have Human Development as our society's official journal. Please note that in addition to accessing e-copies of the journal Human Development, all individual members of JPS may optionally subscribe to the print edition of the Periodical at a reduced annual rate of $\$ 200$ US incl. postage and handling. Our valued connection to Human Development over the last couple of years has yielded important special issues of invited programs from our annual conferences, including the current release of Volume 64 (No. 4-6). This special issue, entitled Globalization, Culture \& Development: Insights and Alternatives in the Contemporary World, was edited by Ashley E. Maynard and Nandita Chaudhary. Drawing on our recent JPS conference, this special issue offers theoretical and empirical studies of the ways that globalization influences human development. Simultaneously, it examines insights about methods aimed at producing more holistic, valid and inclusive accounts of development in the context of globalization, worldwide. This volume beautifully illustrates multiple pathways of development, and stresses that in order to understand those pathways we must understand developmental contexts and their implications for development.

We are in the final stages of organizing JPS's next conference, celebrating Constructivism and the 50th Anniversary of JPS, to be held Tuesday, June 1 through Saturday, June 5, 2021. The conference will be virtual, and registration is $\$ 50$ for regular members and $\$ 30$ for emerging and emeritus scholars. Please also note that members from developing nations receive a $50 \%$ discount. The first day of our upcoming conference will be devoted to understanding the historical presence of JPS and will offer professional development opportunities for emerging scholars and others. Our exciting line-up of plenary speakers in the days to follow will include Susan Goldin-Meadow, Melanie Killen, Cintia Rodríguez, and Tania Zittoun. Please visit our website to learn more about the conference program and its content.

We are continuing to develop our member benefits beyond access to Human Development, as well as a myriad of ways to honor our members' scholarship. Recently, we launched two new JPS awards to be presented annually: the Early Career Award in Developmental Science and the Award for Distinguished Contributions to Developmental Science. In addition, JPS will officially launch a new podcast series How Ideas Travel at our next conference. These podcasts, led by Colette Daiute and Nancy Budwig, highlight conversations with preeminent developmental scholars about the nature, culture, and dynamics of ideas fueling the development of individuals and societies. Through explorations of the personal and interpersonal dimensions of knowledge and knowing, this podcast series reveals how ideas are alive and practical. For anyone interested in how ideas travel and in developing ways to encourage students to consider social and historical aspects of the development of knowledge, please be sure to visit our website for more information. 


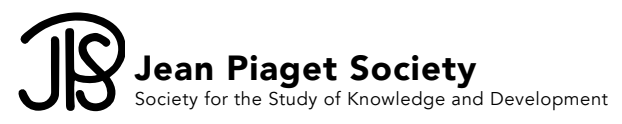

We are thrilled to collaborate with Susan Rivera, Editor of Human Development, as well as members of the Karger team. We hope that all readers of Human Development will take a moment to consider joining the Jean Piaget Society for the Study of Knowledge and Development and consider attending our 50th anniversary conference celebration in June 2021. This new issue of Human Development contains articles by some longtime JPS members, including both the current president of the society, David Witherington, and a past president, Nancy Budwig. We welcome the readership of Human Development to continue discussions of important issues pertaining to the study of human development with us. 